\title{
Analysis and Comparison of EZW, SPIHT and EBCOT Coding Schemes with Reduced Execution Time
}

\author{
Pooja Rawat \\ Scholars of M.Tech \\ GRD-IMT, Dehradun
}

\author{
Arti Rawat \\ Scholars of M.Tech \\ U.T.U., Dehradun
}

\author{
Swati Chamoli \\ Assistant Professor \\ GRD-IMT, Dehradun
}

\begin{abstract}
In the digital era of communication it is very common to sending some information from one point to another. In every field of engineering that is biomedical, astronomical, geological etc. Image is one of the commonly used multimedia. So for fast and efficient communication formulate, image compression is needed in each and every field. Intended for coding of transformed image, here is a comparison between various parameters of three of coding schemes EZW, SPIHT and EBCOT. After the transformation, those coding scheme basically code high energy components first and progressively transmits the coded bits to make an increasingly update and refined copy of the original image. In this paper reduced the execution time and provide the best reconstructed image with higher PSNR by using those coding schemes. The compared results of various parameters of image compression algorithms analyzed using MATLAB software and wavelet toolbox.
\end{abstract}

\section{Keywords}

Image Compression, EZW, SPIHT, EBCOT PSNR, CR, BPP, MSE, execution time.

\section{INTRODUCTION}

In today's digital world transmission and usage of image cannot be avoided. Image compression is one of the technologies which consist memory storage and transmission of images more practical [2] Image Compression is an important technique for making an image file sizes of manageable and transmittable dimensions. The basic need for maintaining the image quality: Transfer rate and Data storage capacity. To achieve a reasonable compression ratio as well as better quality of reconstructed image and high PSNR by using EZW, SPIHT and EBCOT coding schemes. EZW is explained in the paper of J.M. Shapiro [4], SPIHT is the coding scheme which is explained by Pearlman and Said [6]. EBCOT is highly scalable compressed bit stream [10]. In the implementation of this thesis it is needed to work on the images as first by using DWT, and then converted into coefficients of higher and lower frequencies components and then EZW, SPIHT, EBCOT coding is applied to get better results on different parameters when compared with each other.

\section{DISCRETE WAVELET TRANSFORM}

\subsection{Introduction of Wavelet Transform}

Easier way to compress, transmit and analyzed many images is Wavelet transform unlike Fourier transform and discrete cosine transform. Wavelet transform consist of small waves of varying frequency and limited time duration that is called Wavelets. Wavelets are the mathematical functions which are generated from the basis function i.e. mother wavelet by scaling and shifting in frequency domain. To resolve the band limited problem in DCT wavelets (small wave) are used. The difference between sine, cosine and short wave are shown in figure1.2. Sine wave and cosine wave goes the range from $-\infty$ to $+\infty$ while wavelet exists for a short interval of time [2]. It can represent a wavelet signal in mathematical expression as:

$f(x)=\sum_{k} \alpha_{\mathrm{k}} \varnothing \mathrm{k}(\mathrm{x})$

Where $\mathrm{k}=$ integer index of function

$\alpha_{\mathrm{k}}=$ coefficients of basic function

$\emptyset \mathrm{k}(\mathrm{x})=$ set of functions

$\varnothing \mathrm{k}$ ( $\mathrm{x}$ ) may be the scaling and shifted version of basic function and it can be related by :

$\emptyset \mathrm{r}, \mathrm{s}(\mathrm{x})=2^{\mathrm{r} / 2} \emptyset(2 \mathrm{rx}-\mathrm{s})$

The wavelet compression methods are better at representing transients, high-frequency components in two-dimensional images by using wavelet transform. By using wavelet transform, wavelets allow the analysis of signals in both time and frequency domain.

\subsection{Discrete Wavelet Transform (DWT)}

In discrete wavelet transform, all the information is concentrated in a small fraction of coefficient and it is easy and efficient to be compressed and this transform removes the blocking effects unlike DCT compression technique. To obtain a multiple level of decomposition discrete wavelet transform can be implemented by passing the signal through a repeated combination of low pass and high pass filters and down sampling operation on low pass branch outputs [3]. In discrete wavelet transform an image is first decomposed into four parts by critically sub sampling horizontal and vertical channels using frequency based sub band filters. These sub bands represented as LLn ,HLn ,LHn ,HHn. LLn - Low pass filter over rows and columns.

HLn - High pass filter over rows and low pass filter over columns.

LHn - Low pass filter over rows and high pass filter over columns.

And HHn - High pass filter over rows and over columns.

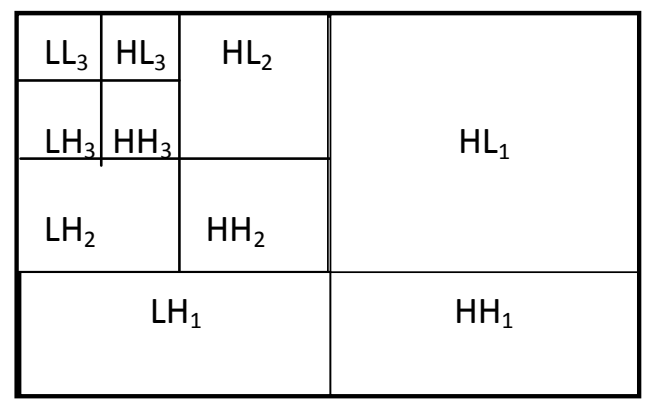

Fig 1: Representation three level wavelet decomposition sub band 


\section{PROPOSED CODING SCHEMES}

\subsection{Embedded Zero tree Wavelet (EZW)}

EZW represents embedded zero tree, the term embedded means encoding the transform magnitudes which allow progressing transmission \& zero-tree is a concept by which compression is achieved to allow for including all the important information of encoding of the position of significant values. The main advantage of this coding is that the encoder can stop the encoding process at any point. The encoding process stop after complete their target. Similarly decoder can decode the lower rate encoding wherever it want and reconstruct the data. Therefore in embedded coding all the pixels can be represented by an embedded bit stream [5].

\subsubsection{Concepts of EZW coding}

- Parent- Child relationship: It is known as ancestordescendant relationship. In this relationship one higher value of coefficient at a coarser scale which represents four coefficients at finer scale. Parent-child relationship means that the coefficient at the coarse scale is parent and at the finer scales all coefficients corresponding to the same spatial location is children [4]. The base of EZW is the parent -child dependencies of sub bands in wavelet transform. For the better performance of zerotree coders parent child relationship responsible.

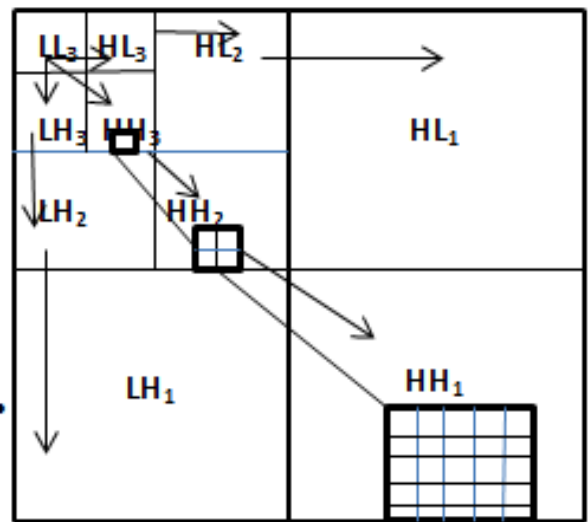

Fig 2: parent-child relation

- Zero trees in EZW: In this concept the root node of wavelet coefficient tree and its corresponding descendants are below the given threshold value then the tree is said to be zero tree and the corresponding root node is known as zero tree root (ZTR).

- Isolated zero in EZW: In this concept the root node of wavelet coefficient tree is less than the given threshold value at any level but if one or more of its descendants are greater than the given threshold then the root is said to be an Isolated zero root.

- Positive and negative significant in EZW: In this concept the absolute value of the wavelet coefficient in the sub-band decomposition of image is greater than the given threshold then it is said to be significant. According to sign of coefficient they are termed as positive significant or negative.

\subsection{EZW Algorithm}

Step1 : This algorithm has first step to set the initial threshold level $\mathrm{T}_{0}=\mathrm{C}_{\max } / 2$. Where $\mathrm{C}_{\max }$ is the maximum coefficient value.

Step2 : Significant Map coding
Dominant pass: In dominant passes coefficients are compared to T0 and their significance map is made. Embedded Zero Tree Wavelet consists of four symbols which actually represents the significant map. In the Dominant pass all the coefficients are tested. If the absolute value of the wavelet coefficient is greater than the given threshold value T0, then the coefficient is said to be significant. Fig. 3 shows the flow chart of dominant pass.

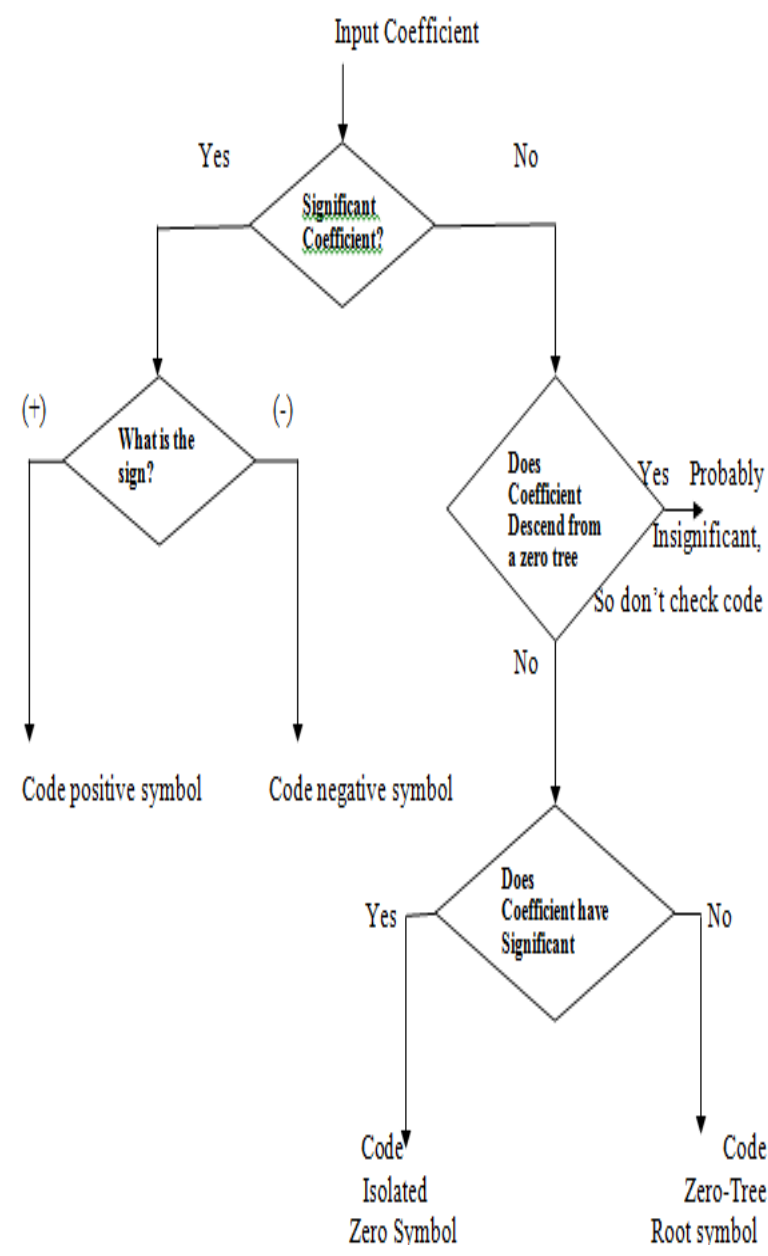

Fig 3 Flow Chart for Dominant Pass Subordinate Pass

The bit streams of symbols which are generated in dominant pass are followed by the subordinate pass. In the subordinate list are refined the value of coefficient. This converts the dominant pass values into bit format if the value is greater than the current threshold then a 1 is sent, if less than the threshold a zero is sent.

Step 3: Now Threshold $\mathrm{T}_{0}=$ threshold $/ 2$ and go to the step 2, when more loops are needed.

\subsection{Set Partitioning In Hierarchal Trees (SPIHT)}

SPIHT algorithm is an improved version of EZW algorithm proposed by A. Said, Pearlman [6]. EZW has two main strengths first one is the embedded bit stream and the second one is the self-similarity between the sub-bands. But EZW have some limitations so that's why advanced version SPIHT used. SPIHT algorithm is based on 3 concepts: Ordered bit plane progressive transmission, set partitioning sorting algorithm, spatial orientation trees [12]. 


\subsubsection{SPIHT Encoding and Decoding}

In SPIHT, this algorithm is identical for encoder and decoder. Both encoder and decoder consists three lists and update continuously. These lists are:
i) List of insignificant pixels (LIP)
ii) List of significant pixels (LSP)
iii) List of insignificant set (LIS)

\subsubsection{Set Partitioning Rules}

Before explaining the rules there are some sets present in spatial orientation tree:

$\mathrm{O}(\mathrm{n} 1, \mathrm{n} 2)$ : This set contains all the coordinates of pixels i.e. offspring of the node $(\mathrm{n} 1, \mathrm{n} 2)$. It is also called as offspring set.

$\mathrm{D}(\mathrm{n} 1, \mathrm{n} 2)$ : It contains the entire pixel coordinate which are descendant of the node $(\mathrm{n} 1, \mathrm{n} 2)$. It is a descendant's set.

$\mathrm{L}(\mathrm{n} 1, \mathrm{n} 2)$ : The difference set between offspring set $\mathrm{O}(\mathrm{n} 1, \mathrm{n} 2)$ and descendant set D (n1, n2). [O (n1, n2) - D (n1, n2)]

$\mathrm{H}$ : This set contains all the spatial orientation tree root's coordinates which belongs to LL sub-band.

Rule 1: Initial Partition consist D (n1, n2) for all $(\mathrm{n} 1, \mathrm{n} 2) \varepsilon \mathrm{H}$

Rule 2: If $\mathrm{D}(\mathrm{n} 1, \mathrm{n} 2)$ found significant then break into $\mathrm{L}(\mathrm{n} 1$, n2) with four single element set $(i, j) \varepsilon O(n 1, n 2)$

Rule 3: If $\mathrm{L}(\mathrm{n} 1, \mathrm{n} 2)$ is found significant then divide it into four set of $D(i, j)$ where $(i, j) \varepsilon O(n 1, n 2)$.

\subsubsection{SPIHT Algorithm}

- Initialization: for output n, LSP is empty; later on add the starting root coordinates to LIP or LIS.

- Sorting Pass: Each entry in LIP, it will decide that it is significant and give the output decision result. If the decision result is significant then moved into the LSP and the coordinate present with their sign. If the rest coordinates all are going to be significant then this process is stopped.

- Refinement Pass: In this pass all the pixel values in LSP are now $2 \mathrm{n} \leq|\mathrm{Ci}, \mathrm{j}|$, and output will be the $\mathrm{nth}$ significant bit.

- Quantization-step update: In this step, decrement $\mathrm{n}$ by 1 and go to step 2.

\subsection{Embedded Block Code With Optimized Truncation (EBCOT)}

EBCOT is an advance technique for image compression. Its earlier processes are EZW and SPIHT coding techniques which are introduced by J.M. Shapiro and Said Pearlman respectively. EBCOT is explained by Taubman [10] obtained for the JPEG 2000 standard. This scheme generates the sub band by using wavelet transform and SNR and resolution scalable compression bit stream. The bit stream which is generated in the output is represented in layers and each layer contains high resolution information of images. Thus decoding of the images is completed layer by layer to achieve a progressive transmission and decomposition.

\subsubsection{Entropy Encoding}

In this entropy encoding firstly image is divided into tiles and tiles is converted into sub band by using wavelet transform and these sub band are quantized into code block and each code block is coded.
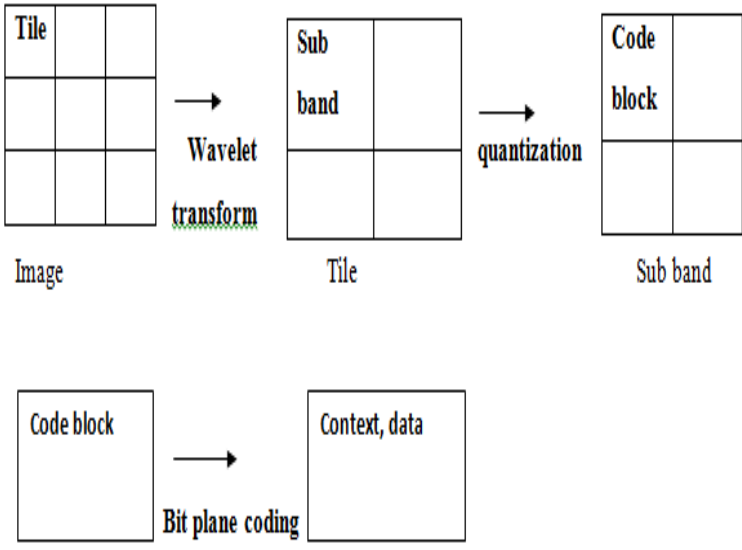

Fig 4 block diagram of entropy coding

For the generation of compressed bit stream [11] is divided into two steps tier1 and tier2.

Tier 1 coding: EBCOT Coder and binary arithmetic coding MQ coder.

\section{i) EBCOT coder}

The basic principle of EBCOT coder is that the EBCOT blocks receives within a code block a set of quantization coefficients together while during the coding process.

\section{ii) Binary Arithmetic Coding MQ-Coder:}

EBCOT produces a sequence of symbols, pair of context and decision during each pass. The block diagram of arithmetic coding MQ coder is shown in fig. 3.9

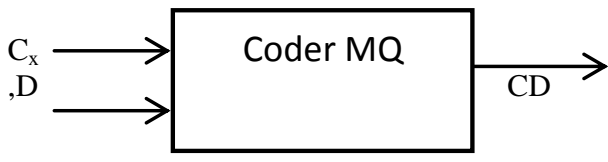

Fig.5 Encoder arithmetic input and output

MQ coder consist the context determined by the neighbor of the bit and the value of symbols and converted into coded bit stream.

Tier 2 coding:

In this tier, the bit stream of code blocks which are generated in tier 1, assemble into packets. These packets are demarcated by header.

There are five permissible progression modes depends on the information which are reconstruct first.

i) Layer resolution component position progression (LRCPP)

ii) Resolution layer component position progression (RLCPP)

iii) Resolution position component layer progression (RPCLP)

iv) Position component resolution layer progression (PCRLP)

v) Component position resolution layer progression (CPRLP)

\subsubsection{EBCOT Algorithm}

EBCOT coder coverts the each bit plane into coded form into the three passes: i) significant propagation pass ii) magnitude refinement pass and iii) cleanup pass. 


\section{i. Significant Propagation Pass}

If the bit location is not significant but at least one of its eight connects neighbor is significant then the bit is coded during in this pass.

\section{ii. Magnitude Refinement Pass}

In this pass all the bits which are coded in the previous pass becomes significant. It includes all the bits from coefficient which are already significant.

\section{iii. Clean Up Pass}

It is the final pass in which all the insignificant coefficients and contain the zero context value are encoded during the process of clean up pass. It is the very first pass for new code block.

\section{RESULT AND ANALYSIS}

In the work here for wavelet decomposition and reconstruction bi-orthogonal 4.4 wavelet was used in the test image and up to three levels decomposition was completed and number of passes of coding was set on five. There are truck test image taken and comparison is done between the result obtained by EZW, SPIHT and EBCOT coding scheme. And comparison is done with respect to different parameters like PSNR, CR, bpp, MSE and execution time.

Gray scale image "truck" size 512 X512 was taken as the test image. Firstly EZW coding was applied on test image after that SPIHT codes and then EBCOT coding and various parameters were measured and PSNR vs pass plot was plotted. And then comparison done between these coding were done. The comparison result got by EZW, SPIHT \& EBCOT is shown in the following Table 1. Figure 6 (a) shows the original image figure $6(\mathrm{~b})$ shows the compressed image by using EZW coding similarly figure 6(c) and figure 6(d) shows the compressed image by using SPIHT and EBCOT simultaneously.

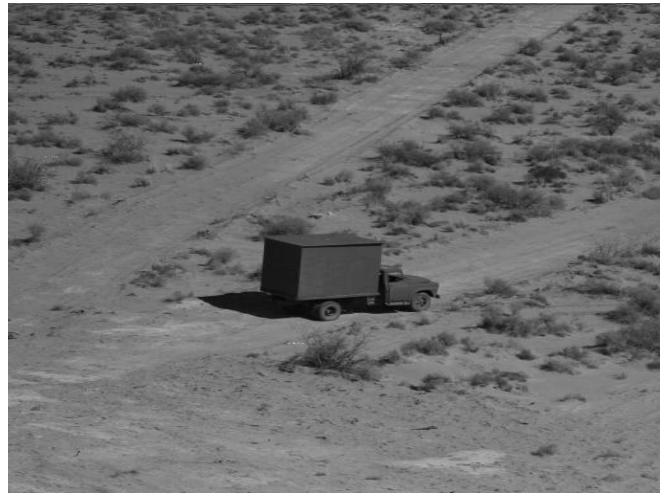

Fig 6(a) Original Image of "Truck"

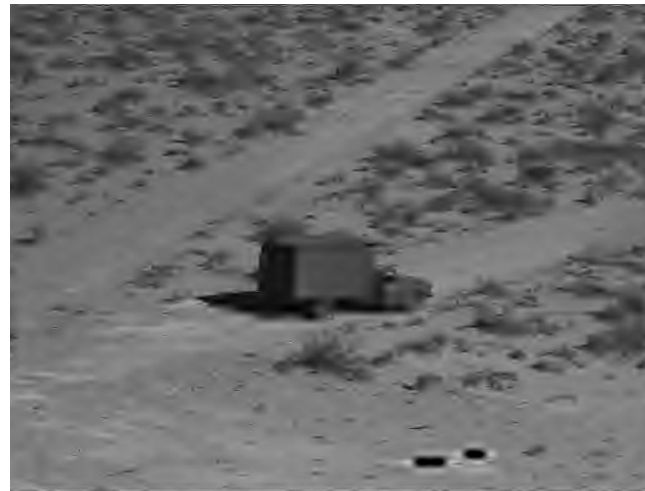

Fig 6(b) Reconstructed Image by EZW

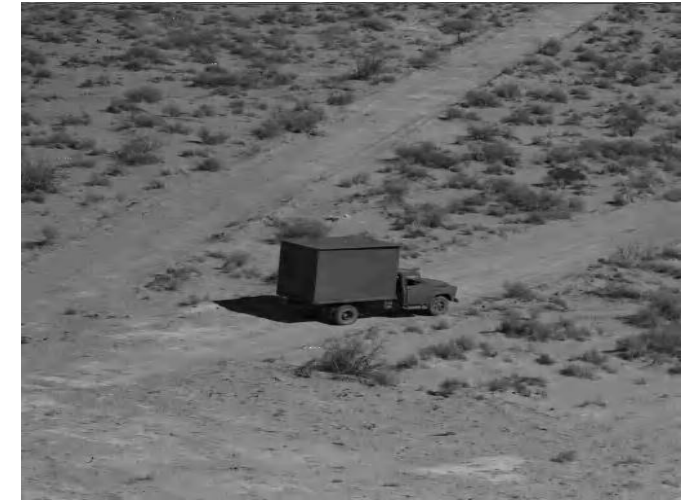

Fig 6(c) Reconstructed Image by SPIHT

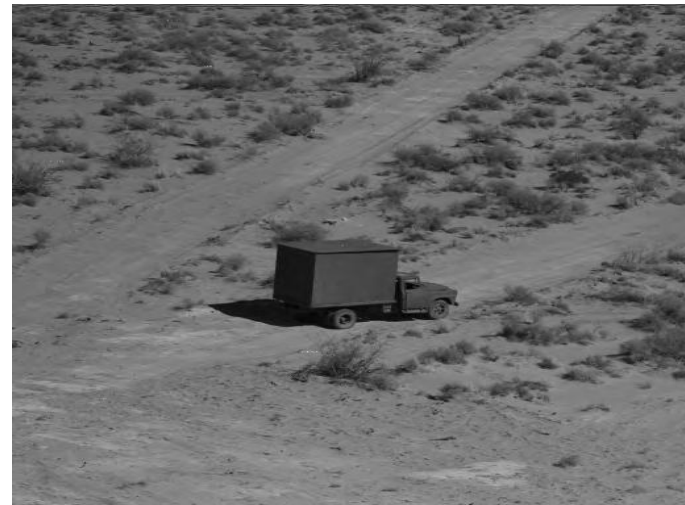

Fig. 6(d) Reconstructed Image by EBCOT

Table 1: Comparison table of EZW, SPIHT and EBCOT Coding Scheme's Parameters

\begin{tabular}{|l|l|l|l|}
\hline Parameters & EZW & SPIHT & EBCOT \\
\hline PSNR (db) & 26.9446 & 33.0494 & 36.9856 \\
\hline MSE & 131.4063 & 32.221 & 13.0173 \\
\hline Bpp & 1.3793 & 1 & 1 \\
\hline CR(\%) & 82.7583 & 87.5 & 87.4999 \\
\hline Rate & 5.7999 & 8 & 7.999 \\
\hline $\begin{array}{l}\text { Execution } \\
\text { time }\end{array}$ & 36.5999 & 23.4974 & 12.7039 \\
\hline
\end{tabular}

The graphical representation of comparision of different parameters by using EZW, SPIHT and EBCOT are given by figure 7(a), 7(b), 7(c), 7(d)and 7(e). 


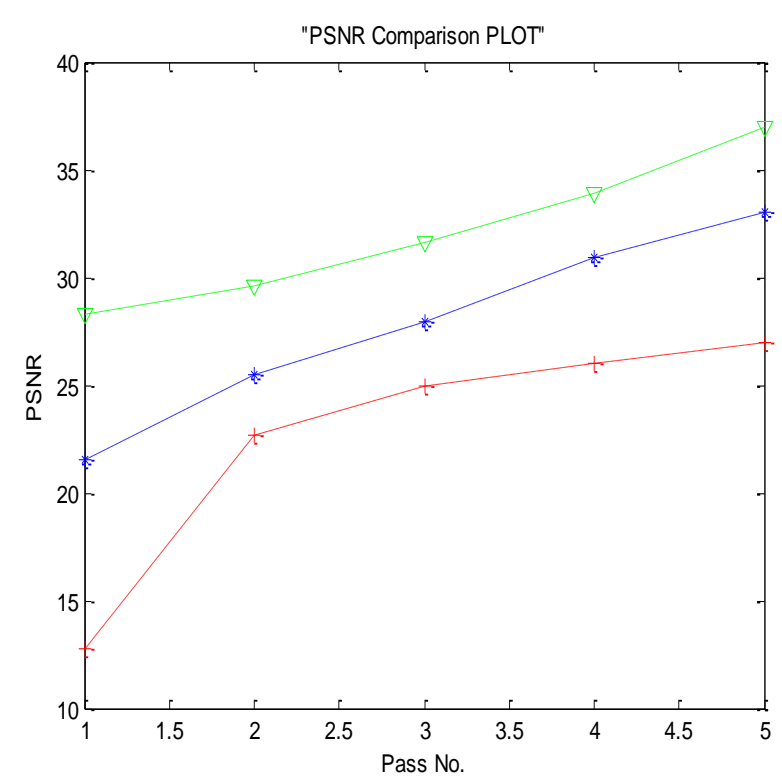

Fig 7(a) PSNR plot for 5 passes between EZW, SPIHT and EBCOT schemes

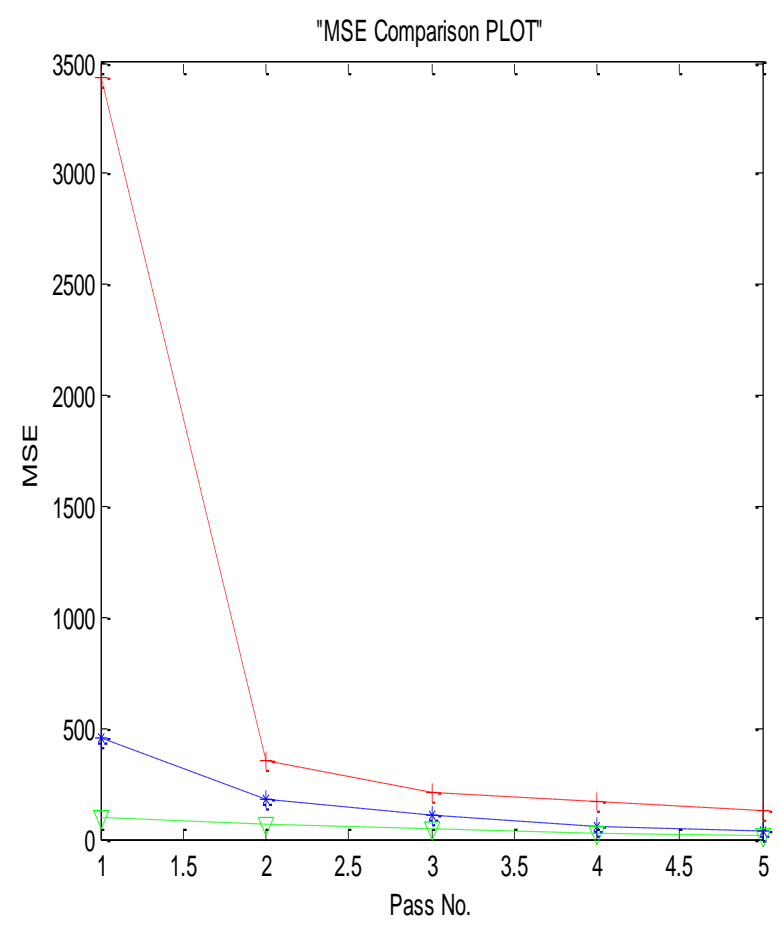

Fig 7(b) MSE plot for 5 passes between EZW, SPIHT and EBCOT schemes

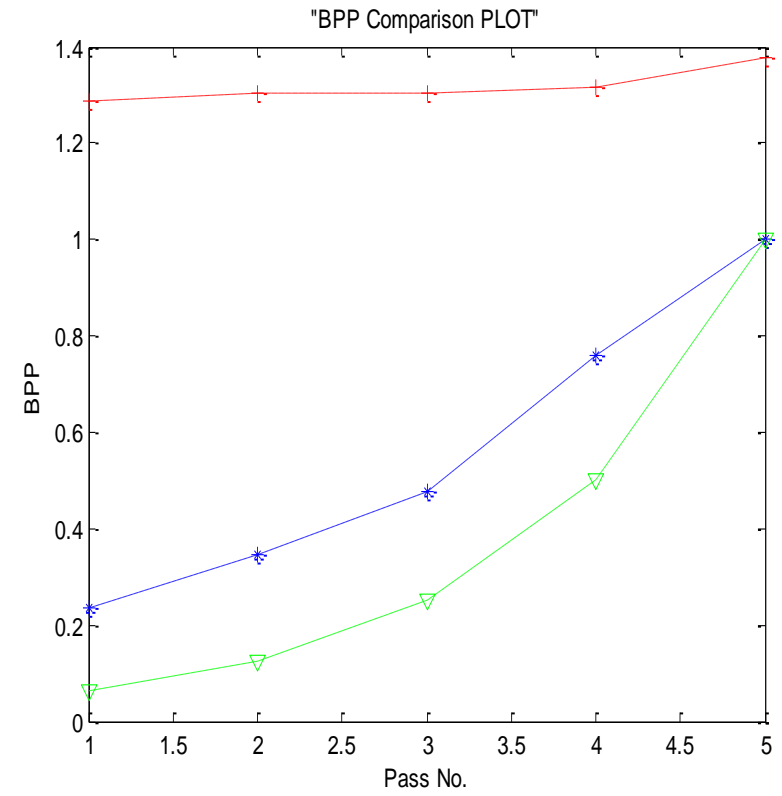

Fig 7(c) bpp plot for 5 passes between EZW, SPIHT and EBCOT schemes

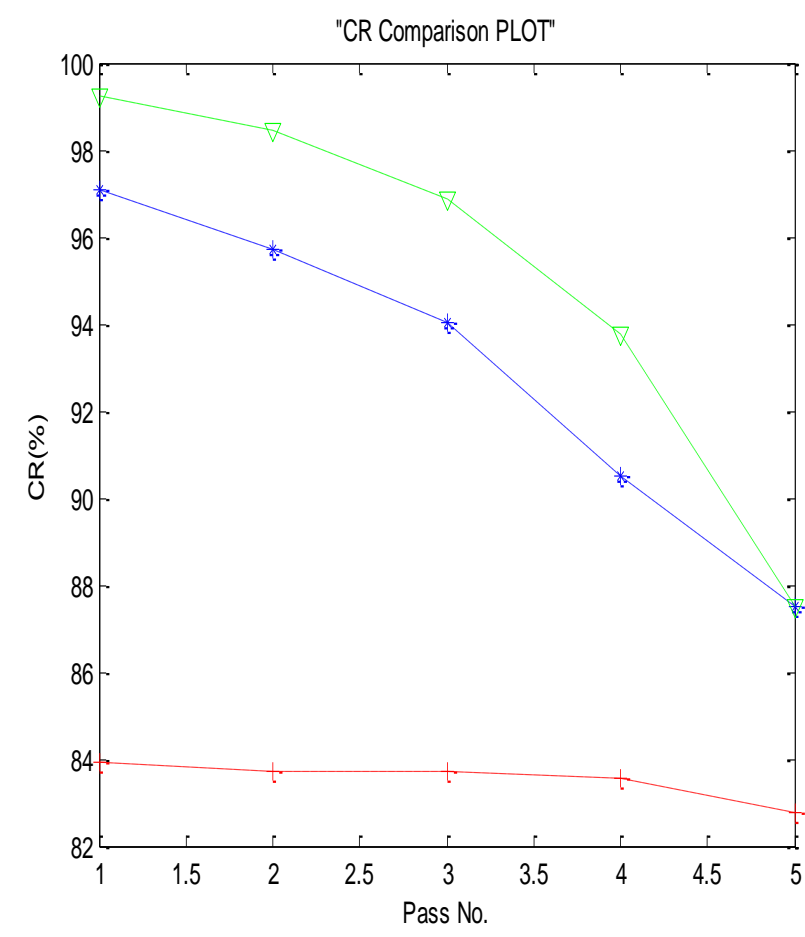

Fig 7(d) CR plot for 5 passes between EZW, SPIHT and EBCOT schemes 


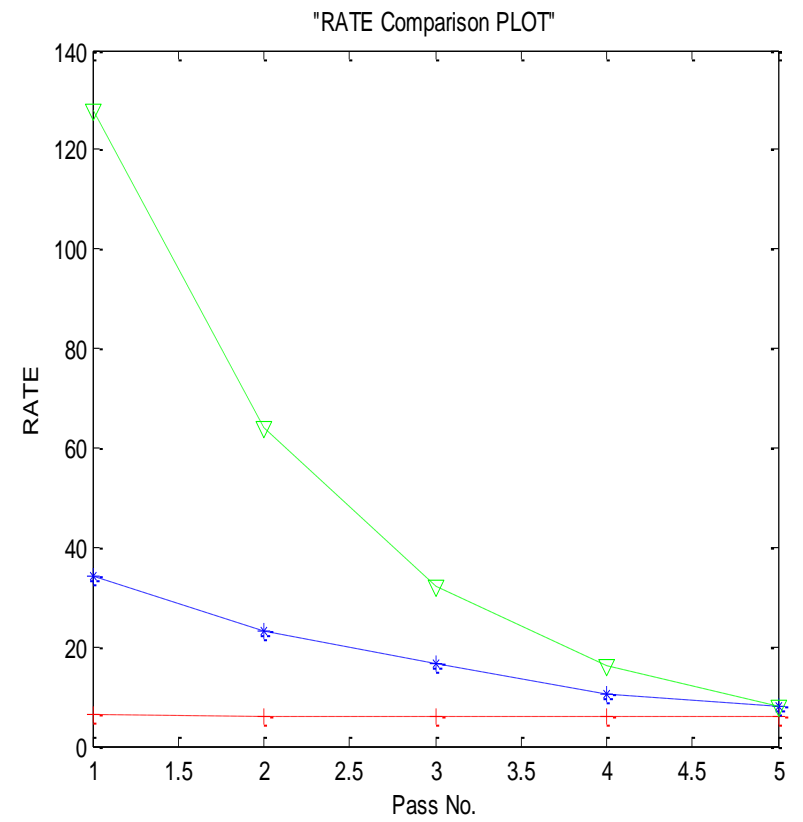

Fig 7(e) CR plot for 5 passes between EZW, SPIHT and EBCOT schemes

\section{CONCLUSION AND FUTURE WORK}

By comparing the numerical value and plots of parameters, EBCOT coding scheme have a better performance. This coding gives a better PSNR less MSE and less execution time for compression of grey scale image.

The results show that the EBCOT coding is very much similar to SPIHT because both are using wavelet transform. But the main difference is SPIHT used the correlation between sub bands while EBCOT encodes the sub band individually.

It has been observed that execution time is become very less for EBCOT coding as compare to EZW and SPIHT coding. So overall EBCOT coding is giving improved results over EZW and SPIHT coding schemes.

In the future work apply the EBCOT coding techniques in the color image compression and in the bio-medical images. Further research on the evaluation of quality in color images is needed to improvisation in peak signal to noise ratio and compression ratio.

\section{REFERENCES}

[1] Soman, K.P., Ramchandran, K.I., 2005, "Insight into Wavelets - From Theory to Practice", Prentice Hall of India, Second Edition, pp. 6-9.

[2] Gonzalez, Rafael C., and Woods, Richard E., 2002 Digital Image Processing. Pearson Education, Englewood Cliffs.
[3] Antonini M., Barlaud M., Mathieu P., Daubechies I.,1992.:"Image Coding Using Wavelet Transforms" IEEE Trans. Image Processing, vol. 1, no. 2, pp 205-220.

[4] Shapiro, J.M., 1993, "Embedded Image Coding Using Zero trees of Wavelet Coefficients" IEEE Trans. on Signal Processing, vol. 41, issue 12, pp 3445-3462.

[5] Raid A.M., .Khedr W.M, El-dosuky M. A. and Ahmed W., 2014, "Image Compression uses Zero tree Wavelet", Signal \& Image Processing: An International Journal (SIPIJ) Vol.5, No.6.

[6] Said A. and Pearlman W. A., 1996, "A new, fast and efficient image codec based on set-partitioning in hierarchical trees," IEEE Trans. Circuits Syst. Video Technol., vol. 6, pp. 243-250 (Jun. 1996).

[7] Dodla S., Y David SolmonRaju, Murali Mohan K.V., 2013, "Image Compression using Wavelet and SPIHT Encoding Scheme", International Journal of Engineering Trends and Technology (IJETT) - Volume 4 Issue 9(Sep 2013).

[8] Singh P., Singh Priti, 2012, "A Comparative Study of Improved Embedded Zerotree Wavelet Image Coder for True and Virtual Images", IEEE Trans.

[9] Zhu L. and Yang Y.M, School of Mechanical Engineering and Automation Wuhan Textile University Wuhan, China; 2011, "Embeded Image Compression Using Differential Coding and Optimization Method", IEEE 978-1-4244-6250-6.

[10] Taubman D.S., "High performance scalable image compression with EBCOT", IEEE Trans. Image Processing, vol. 9, pp. 1158-1170, July 2000.

[11] Medouakh S. and Baarir Z.E.,2011, "Entropy Encoding EBCOT (Embedded Block Coding with optimized Truncation) in JPEG 2000", IJCSI, vol. 8, issue 4, no.1, july 2011 .

[12] Nautiyal A., Tyagi I. and Pathela M., 2014, "PSNR Comparison of Lifting Wavelet Decomposed Modified SPIHT Coded Image with Normal SPIHT Coding" International Journal of Computer Applications 102(15):16-21.

[13] Singh P., Singh Priti; 2011, "Design and Implementation of EZW \& SPIHT Image Coder for Virtual Images", IJCSS, vol. 5 , issue 5 .

[14] Gupta R., Joshi M. 2015, “Gray Scale Image X-ray Image Compression using Block Truncation Coding Technique", IJARCSSE, vol 5 issue 5, May 2015.

[15] Nautiyal A., Tyagi I., Bijalwan V., Balodhi M., 2014, "Enhanced EZW Technique for Compression of Image by Setting Detail Retaining Pass Number", arXiv preprint arXiv: 1407.3673. 